\title{
Gesprächspsychotherapie mit PatientInnen mit einer Borderline-Persönlichkeitsstörung
}

\author{
Jochen Eckert · Eva-Maria Biermann-Ratjen
}

Online publiziert: 21. Oktober 2020

(c) Der/die Autor(en) 2020

\begin{abstract}
Zusammenfassung Das zentrale Therapeutikum der Gesprächspsychotherapie ist ihr Beziehungsangebot: Der/die TherapeutIn bemüht sich, die PatientIn in ihrem Erleben bedingungsfrei positiv zu beachten und empathisch genau zu verstehen. Dieser Beitrag beschreibt und erläutert, wie sich die Symptomatik des/r Borderline-PatientIn, die vor allem Ausdruck einer Beziehungsstörung ist, auf die Möglichkeiten des/r PatientIn auswirkt, dieses Beziehungsangebot wahr- und anzunehmen.

Es wird dargestellt, wie der/die GesprächspsychotherapeutIn damit umgehen kann und dass ein/e TherapeutIn, die eine/ $n$ Borderline-PatientIn in Therapie nimmt, sich auf eine krisenanfällige Langzeitbehandlung einstellen sollte.

Der Beitrag schließt mit Empfehlungen zum therapeutischen Rahmen bzw. Versorgungsaspekten.
\end{abstract}

Schlüsselwörter Borderline-Persönlichkeitsstörung · Gesprächspsychotherapie · Therapeutische Rahmenbedingungen

\section{Person-centered psychotherapy with patients} with a borderline personality disorder

Summary The core of personcentered psychotherapy is the therapeut's offer of a specific relationship: He

Für das von Carl R. Rogers begründete

Psychotherapieverfahren ist in Österreich und in der Schweiz die Bezeichnung „Personzentrierte Psychotherapie“ (PZT) und in Deutschland die Bezeichnung „Gesprächspsychotherapie“ (GPT) gebräuchlich. Wir verwenden im vorliegenden Text die Bezeichnung GPT synonym mit PZT.

\section{J. Eckert $(\square)$}

Loehrsweg 1, 20249 Hamburg, Deutschland

eckert.biermann-ratjen@t-online.de tries to unconditionally positively regard and empathically understand whatever the patient is presently experiencing.

This article describes and illustrates the diffculties of Patients with a Borderline Personality Disorder-whose symptoms are foremost problems in establishing and living in close relationships-to realize and accept this therapeutic offer. It exemplifies how the therapeut can handle these diffculties and demonstrates that psychotherapy with a Borderline Patient means a longtime relationship full of critical events.

The article closes with recommendations concerning the therpeutic frame and aspects of health care.

Keywords Borderline personality disorder · Personcentered psychotherapy · Therapeutic framework conditions

\section{Einleitung}

In einem vor über zehn Jahren erschienenen Lehrbuch der Psychotherapie, in dem auch die verschiedenen Behandlungsansätze für PatientInnen mit einer Borderline-Persönlichkeitsstörung dargestellt werden (Dulz und Eckert 2007), kommen die Autoren zu dem Schluss:

Bleibt für Psychotherapeuten die Erkenntnis: Borderline-Patienten kann man nur selten mit nur einer einzigen Behandlung umfassend und dauerhaft helfen. In der Regel können wir ihnen eine Hilfe geben, die eine Zeit lang trägt, und manchmal besteht unsere Hilfe darin, dass wir einräumen müssen, ihnen nicht bzw. nicht ausreichend helfen zu können (a.a. O., S. 594).

Die Feststellung, dass die Behandlung von PatientInnen mit einer Borderline-Persönlichkeitsstörung 
zeitaufwändig ist, auch wegen notwendiger Behandlungswiederaufnahmen, und dass darüber hinaus sich der Behandlungserfolg vergleichsweise in Grenzen hält, gilt auch heute noch.

Die Gründe dafür sind, dass die psychische Entwicklung dieser Personen früh gestört ist, dass sie kein stabiles Selbst ausbilden konnten und dass ihre Symptomatik im Laufe ihrer Entwicklung wechselt und sie nicht nur die unterschiedlichsten neurotischen sondern auch psychotische Symptome entwickeln.

Da der Heilungsprozess bei dieser Störung nicht in erster Linie im Erkennen und Bewältigen unbewusster Konflikte besteht bzw. im Korrigieren von Fehlanpassungen, sondern im Erwerb eines ausreichend stabilen Selbst, erfordert er einen größeren Zeitaufwand.

Während es durch die Festschreibung der Kriterien für die Diagnose Borderline-Persönlichkeitsstörung in den psychiatrischen Klassifikationssystemen DSM-5, ICD-10 (F) und durch den Einsatz von Diagnostischen Interviews (z. B. Diagnostisches Interview für Borderline-Patienten („DIB“), s. Eckert et al. 2005) zu einer Vereinheitlichung bei der Diagnosestellung gekommen ist, sind die Ursachen für die Störung weiterhin strittig. Die Annahme, dass es sich bei der Borderline-Persönlichkeitsstörung um eine subschizophrene Störung handelt, wird heute kaum noch vertreten. Diskutiert wird derzeit vor allem, ob es sich um den Ausdruck einer subaffektiven Störung oder um eine Impulskontrollstörung oder um eine Posttraumatische Belastungsstörung handeln könnte (BiermannRatjen et al. 2019; vgl. auch den Beitrag von Dulz in diesem Heft).

\section{Therapietheoretische Grundannahmen der Gesprächspsychotherapie (GPT)}

Im Zentrum der von dem amerikanischen Psychologen Carl Rogers entwickelten Therapietheorie (Rogers 1959; s. a. Biermann-Ratjen et al. 2016) steht die therapeutische Beziehung:

Entwickelt sich zwischen TherapeutIn und PatientIn eine therapeutische Beziehung dergestalt, dass der/die PatientIn zumindest im Ansatz wahr- und annehmen kann, dass ihn/r die TherapeutIn bedingungsfrei positiv beachtet und im Erleben empathisch versteht, wobei der/die TherapeutIn selbst kongruent ist, ermöglicht das dem/r PatientIn, sich dem eigenen Erleben und dessen Bewertungen reflektierend zuzuwenden, sie zu erkennen, zu benennen und gemeinsam mit dem/r TherapeutIn zu betrachten. Dadurch wird es ihm/r möglich, bisher verzerrte bzw. abgewehrte Erfahrungen und deren Bewertung durch den/die PatientIn selbst zuzulassen und in das SichSelbst-Erleben bzw. Selbstbild zu integrieren.

Wir werden im Folgenden aufzeigen, dass genau das die Symptomatik der Borderline-PatientInnen im Kern ausmacht: die Unfähigkeit dieses Beziehungsangebot anzunehmen und mit Unterstützung des/r TherapeutIn sich dem ganz eigenem Erleben und dessen
Bewertung durch sich selbst und in Bezug zum eigenen Selbstbild zuzuwenden.

\section{Die borderline-typische Symptomatik und ihre Auswirkungen auf die Gestaltung persönlicher und psychotherapeutischer Beziehungen}

Die Symptomatik von PatientInnen mit einer Borderline-Persönlichkeitsstörung wird im DSM-5 (Falkai und Wittchen 2018) zusammenfassend so charakterisiert: „Ein tiefgreifendes Muster von Instabilität in zwischenmenschlichen Beziehungen, im Selbstbild und in den Affekten sowie von deutlicher Impulsivität. Der Beginn liegt im frühen Erwachsenenalter, und das Muster zeigt sich in verschiedenen Situationen“. (DSM-V 2018, S. 908). D.h. diese PatientInnen entwickeln nicht nur Symptome in unterschiedlichen Bereichen, nämlich in denen der Affekte und Kognitionen, in der Impulskontrolle und in den zwischenmenschlichen Beziehungen, sondern auch zu einer Zeit in dem einen und $\mathrm{zu}$ einer anderen in dem anderen Bereich und mit unterschiedlicher Intensität. RohdeDachser hat das in dem Satz zusammengefasst, dass das Stabilste an der Symptomatik der PatientInnen mit einer Borderline-Störung die Instabilität sei.

Bestimmte borderline-spezische Symptome, z.B. ihr psychotisches Erleben, legen die Erklärung nahe, dass diese PatientInnen kein ausreichend stabiles Selbstkonzept haben, sondern eines, das unter starker psychischer Belastung seine realitätsprüfenden Funktionen verliert, so dass dissoziative Zustände auftreten oder z. B. Depersonalisations- oder Derealisationszustände. Borderline-PatientInnen erleben sich auch oft als existentiell bedroht in der Überzeugung, so abgrundtief böse zu sein, dass sie keine Existenzberechtigung haben und jeder andere Mensch, der das erkennt, sich von ihnen abwenden, sie wegschicken oder verlassen wird. Die Vermeidung des Kontaktes mit dieser Überzeugung spielt oft auch bei der bei Borderline-PatientInnen vermehrt zu beobachtenden „Spaltung“ eine Rolle. Die PatientInnen retten sich z. B. in die Überzeugung, dass die Person, von der sie sich total entwertet wähnen, die eigentlich Böse ist und sie deren Opfer.

M. Mahler (1972, zit. nach Rohde-Dachser, 1995, S. 78) hat vorgeschlagen, bei den von Borderline-PatientInnen eingesetzten Strategien zur Abwehr von unerträglichen, weil die eigene Existenz infrage stellenden Erfahrungen nicht von Abwehr- sondern von Erhaltungsmechanismen zu sprechen.

Bestimmte dieser Erfahrungen, wie die einer inneren Leere oder die Vernichtungsängste, sind TherapeutInnen außerhalb des therapeutischen Kontaktes in aller Regel selbst nicht zugänglich. Sie vermitteln sich ihnen aber im therapeutischen Kontakt und sind nicht leicht auszuhalten. Der/die (Gesprächs)PsychotherapeutIn sollte selbstreflexiv darauf achten bzw. sich bewusst machen können, ob er/sie sich derart „angesteckt“ hat (vgl. Eckert et al. 2012, S. 182). 
In Behandlungsteams identifizieren sich einzelne Mitglieder oft mit bestimmten Aspekten des Erlebens ihrer Borderline-PatientInnen, was zu massiven Teamkonflikten führen kann.

\section{Affekte}

Ähnliches gilt für Wut und Ärger, Gefühle unter denen PatientInnen mit einer Borderline-Störung häufig chronisch leiden. Sie können Wut und Ärger vor allem nicht als ihr Erleben akzeptieren. Tauchen sie auf, ist das für sie der Beleg dafür, dass sie abgrundtief schlecht sind und es verdienen, abgelehnt und weggeschickt bzw. verlassen zu werden.

In einer Gruppensitzung wurde ein Borderline-Patient von einem anderen Gruppenmitglied massiv attackiert und beleidigt. Alle in der Gruppe bekamen mit, dass der Attackierte angestrengt versuchte, nicht wütend zu werden. Als die Gruppentherapeutin das ansprach (,,das was Herr X ihnen gerade vorgeworfen hat, macht sie ganz schön sauer?!") wurde der Angesprochene leichenblass, um dann in einem resignierten Ton zur Gruppe zu sagen: „Ich weiss ja, dass ihr mich alle für ein mieses Schwein haltet".

Diese PatientInnen versuchen deshalb diese Gefühle zu vermeiden, z. B. indem sie ihnen wichtige Bezugspersonen idealisieren.

Viele Borderline-PatientInnen entwickeln in Bezug auf ihr Wuterleben die Angst, die Kontrolle über ihr Verhalten $\mathrm{zu}$ verlieren und nur noch zerstörerisch agieren zu können.

So reagierte ein Borderline-Patient in einer Gruppensitzung auf den Vorschlag eines Gruppenmitglieds, doch unter eine Eisenbahnbrücke zu gehen, und wenn ein Zug sie überquert, seine Wut laut herauszuschreien, fast panisch: Wenn er seine Wut zulasse, dann sähe er sich mit einem Maschinengewehr die Mönckebergstraße (eine belebte Hamburger Einkaufsstraße) entlanggehen und alles niederknallen, was ihm in den Weg kommt.

Für den/die PsychotherapeutIn folgt daraus, nicht den vorliegenden Wutaffekt zu thematisieren, sondern das ganze Erleben, vor allem die Angst des/r PatientIn vor Wut und Ärger.

Borderline-PatientInnen klagen häufig über depressive Gefühle. Dabei handelt es sich meisten nicht um Reaktionen auf herbe Enttäuschungen oder Verluste, sondern um dysphorische Zustände, wie Freudlosigkeit oder das Gefühl innerer Leere.

Während in Gruppentherapien mit PatientInnen mit neurotischen Störungen häufiger auch mal gescherzt, gelacht und geflirtet wird, herrscht in Gruppen mit Borderline-PatientInnen oft ein Klima, das gekennzeichnet ist durch ängstliches Schweigen und eine Gespanntheit wie vor einer drohenden Katastrophe.
Das Handeln des/r GesprächspsychotherapeutIn beschränkt sich am besten auf den Versuch, die Gefühle, die die Situation bestimmen, vorsichtig $\mathrm{zu}$ benennen, sie mit den PatientInnen zusammen auszuhalten und sich von ihnen nicht „anstecken“ zu lassen. Bei der Thematisierung dieser Gefühle geht es häufig vor allem um Strategien, die PatientInnen für sich entwickeln, solche dysphorischen Zustände $\mathrm{zu}$ überwinden, z.B. die innere Leere mit Schmerz zu bekämpfen, wozu das Schneiden bzw. Ritzen der Unterarme ein probates Mittel zu sein scheint.

\section{Kognitionen}

PatientInnen mit einer Borderline-Persönlichkeitsstörung berichten häufig von wiederkehrenden Sinnestäuschungen.

Wenn ich allein in einem Zugabteil sitze und jemand zusteigt und sich auch in meinem Abteil niederlassen will, habe ich das sichere Gefühl, dass das nicht geht, weil sich mein Körperumfang verdreifacht hat. Ich weise den Fahrgast dann darauf hin, dass er doch sehen muss, dass hier für ihn kein Platz ist.

Fast regelhaft berichten PatientInnen von Depersonalisationserfahrungen. Sie fühlen sich fremd, können sich nicht spüren, fühlen sich von ihren Gefühlen getrennt. Sie versuchen dann, den Kontakt zu sich selbst wieder herzustellen, z. B. indem sie sich kneifen oder sich in einem Spiegel betrachten.

Tritt dieses Fremdheitserleben in Bezug auf Gegenstände oder auf die vertraute Umgebung auf, spricht man von Derealisationserlebnissen.

Diese Wahrnehmungserlebnisse haben häufig nur mittelbare Auswirkungen auf den therapeutischen Kontakt. Sie treten eher dann auf, wenn der/die PatientIn alleine ist.

Eine Patientin berichtete, dass sie in eine U-Bahn gestiegen sei, um einen Termin bei ihrem behandelnden Psychiater wahrzunehmen. Da sei sie aber nie angekommen. Vielmehr sei sie von der Polizei als "hilflose Person“ an der Endstation der $U$-Bahn in Gewahrsam genommen worden. Sie habe nicht mehr gewusst, dass sie auf dem Weg zu ihrem Psychiater gewesen war. Es stellte sich dann heraus, dass dieser ihr bei ihrem letzten Besuch den Wunsch nach häufigeren Kontakten abgeschlagen hatte.

$\mathrm{Zu}$ den Symptomen im Bereich Kognitionen gehören auch vorübergehende paranoide Erlebnisse, die aber nicht unkorrigierbar wahnhaft sind, sondern an deren Realitätsgehalt die PatientInnen meistens selbst zweifeln (,ich kann mir aber auch nur einbilden, dass man hinter meinen Rücken über mich gelacht hat") und die nicht andauernd sind.

Der/die GesprächspsychotherapeutIn sollte davon absehen, mit dem/r PatientIn klären $\mathrm{zu}$ wollen, ob 
es sich bei den von dem/r PatientIn als bedrohlich geschilderten Erfahrungen um realitätsentsprechende oder eingebildete Wahrnehmungen handelt. Er/sie sollte vielmehr mit dem/r PatientIn zusammen versuchen zu erfassen, was diese Erfahrungen für dessen/ deren Sich-selbst-Erleben bedeuten.

\section{Impulshandlungen}

Impulskontrollverluste gehören zur borderline-typischen Symptomatik und führen nicht selten zu einer stationären psychiatrischen Aufnahme, wenn es z.B. zu körperlichen Selbstbeschädigungen oder gravierenden Suizidversuchen kommt.

$\mathrm{Zu}$ den Impulshandlungen gehört der Substanzmissbrauch, der sich auf alle denkbaren rauscherzeugenden Substanzen, u.a. Drogen, Medikamente und Alkohol, erstreckt. Die Substanzen wechseln, meistens ist nicht die Art der Substanz, sondern ihre akute Verfügbarkeit wichtig. Es bleibt meistens beim episodischen Abusus, ein chronischer Abusus einer bestimmten Substanz entwickelt sich typischerweise nicht.

Impulshandlungen während eines therapeutischen Kontaktes sind nach unserer Erfahrung eher selten, aber nicht ausgeschlossen.

Ein Beispiel dafür ist eine aus der stationären Psychotherapie entlassene Patientin. Sie nahm an einer Nachbehandlungsgruppe teil und war auch noch krankgeschrieben. Sie wandte sich telefonisch an ihren Therapeuten und beklagte sich bitterlich, dass ihre Mutter von ihr verlange, ihre Berufstätigkeit wieder aufzunehmen. Sie sei doch noch krankgeschrieben und fühle sich dazu überhaupt nicht in der Lage. Ob man denn nicht gerichtlich die Mutter dazu zwingen könne, diese Forderungen zu unterlassen.

Der Therapeut schlug vor, zusammen mit der Mutter zu einem klärenden Gespräch zu kommen. Zwei Tage später erschienen Mutter und Tochter (Patientin) zu diesem Gespräch. Der Therapeut bat zunächst die Patientin ohne die Mutter in das Behandlungszimmer, wo die Patientin voller Wut und Empörung die Forderungen der Mutter wiederholte, die zu erfüllen sie sich nicht imstande fühlte. Der Therapeut bat daraufhin die Mutter hinzuzukommen und wandte sich mit den Worten an sie: „Ihre Tochter hat mir erzählt... “. Weiter kam er nicht, weil die Tochter voller Wut den Therapeuten anschrie, was er sich denn dabei denke, ihre Mutter schlecht zu machen. Ihre Mutter habe Recht: Er sei doch an allem Schuld. Wenn er ein guter Therapeut wäre, dann könnte sie doch jetzt auch wieder arbeiten.

Voller Zorn sprang sie auf, riss ihren Stuhl hoch und schmetterte ihn aufden Boden. Laut schimpfend auf unfähige Therapeuten stürmte sie aus dem Behandlungszimmer, räumte im Flur noch die Topfblumen von einem Fensterbrett ab, um dann aus der Klinik zu stürmen.

Ein solches Verhalten könnte dazu führen, dass der Therapeut die Behandlung nicht fortsetzt, da die Patientin sein therapeutisches Engagement für sie ja offenbar nicht wahrnehmen kann; sie entwertet ihn und seine therapeutischen Bemühungen total.

Auf der anderen Seite ist es aber so, dass der Therapeut die Patientin möglicherweise nicht versteht. Das Verstehen der Reaktion dieser Patientin z. B. stellt sich - wie oft und bei vielen borderline-spezifischen Symptomen und Verhaltensweisen - nicht durch Einfühlung ein. Der Therapeut kann aber auf der Grundlage seines Wissens um borderline-spezifische Formen der Abwehr, im geschilderten Fallbeispiel durch Wissen um das Phänomen der Spaltung, erschliessen: Der Patientin ist es nicht möglich, die Enttäuschung über ihre Mutter in deren Gegenwart zu spüren. Wenn sie sich dem Gefühl der Enttäuschung nähert, taucht die Angst vor Trennung auf, z.B. von der Mutter weggeschickt zu werden. Da die Patientin zu Recht annimmt, dass der Therapeut die Mutter darauf hinweisen wird, dass sie mit ihrer Forderung der Wiederaufnahme der Berufstätigkeit die Tochter überfordert, richtet sich ihre Wut auf ihn. Er ist es nun, der die Mutter verbotenerweise kritisiert. Wenn dem Therapeuten z. B. in einer Supervision diese Zusammenhänge deutlich werden, kann er sich auch vorstellen, die therapeutische Arbeit mit dieser Patientin fortzusetzen. Das war in diesem Fall nur dadurch möglich, dass er den Kontakt zu der Patientin von sich aus wieder aufnahm und ihr einen Gesprächstermin anbot. Die Patientin war nach ihrem Ausbruch - wie zu erwarten (s. oben) - davon ausgegangen, dass nun der Therapeut sie nie wieder sehen wollte.

Auch Suizidversuche während einer Behandlung bedrohen in der Regel die therapeutische Beziehung. TherapeutInnen könnten Suizidhandlungen ihrer PatientInnen als persönliches Versagen verbuchen oder als Bruch eines mit dem/r PatientIn vereinbarten „Suizidvertrages“ mit der Konsequenz, dass sie die Behandlung für beendet erklären.

Manche Suizidhandlungen lassen sich aber auch als Belastungsprobe für die therapeutische Beziehung verstehen. Sie sollten unter allen Umständen wie jedes andere Symptom Thema der Therapie werden.

Sollte der Suizidversuch zu einer stationären Aufnahme führen, ist es im Hinblick auf die bestehende Trennungsproblematik der PatientInnen erforderlich, dass der/die TherapeutIn den Kontakt aufrechterhält, z. B. brieflich oder durch einen kurzen Krankenbesuch.

\section{Zwischenmenschliche Beziehungen}

Tab. 1 fasst typische Belastungen der therapeutische Beziehung bei der Arbeit mit PatientInnen mit einer 
Tab. 1 Problematische borderline-typische Beziehungsmuster und Reaktionen in einer therapeutischen Beziehung

Aktive Beziehungsmuster
Der Patient idealisiert und entwertet uns
Er lähmt uns mit seiner inneren Leere und fehlendem Zugang zu seinem
eigenen Erleben
Er vermeidet aktiv wirklichen Kontakt, meistens aus Angst vor Nähe und
den damit für ihn verbundenen Gefühlen von Ohnmacht sowie der Angst,
verlassen zu werden
Er reagiert auf auf die Verbalisierung von einfühlendem Verstehen, wenn
diese sich auf Gefühle und Erfahrungen bezieht, die nicht mit seinem
Selbstkonzept im Einklang steht, mit heftiger Abwehr. Er fühlt sich dann
nicht verstanden, sondern bedroht
Vermeidende Beziehungsmuster
Der/die Borderline-Patientln dissoziiert
Er wird psychotisch
Er wird suizidal
Er bleibt der Therapie fern, d. h., er bricht sie ab

Borderline-Persönlichkeitsstörung zusammen (Eckert 2018, S. 242).

Wir wiederholen an dieser Stelle: Der zentrale therapeutische Faktor der Gesprächspsychotherapie ist eine TherapeutIn-PatientIn-Beziehung, in der ein/e PatientIn wahr- und annehmen kann, dass die kongruente TherapeutIn ihn/sie in den Erfahrungen und deren Bewertungen empathisch versteht und bedingungsfrei positiv beachtet.

Es ist unmittelbar evident, dass die in der Tab. 1 aufgelisteten problematischen Beziehungsmuster vor allem die „Bedingungsfreie positive Beachtung“ des/r GesprächspsychotherapeutIn für den/die PatientIn gefährden. Es ist für die meisten TherapeutInnen nicht leicht auszuhalten, wenn sie von ihrem/r PatientIn plötzlich und unerwartet entwertet werden und wenn das auch noch öffentlich geschieht, z. B. in einer Gruppensitzung wie im folgenden Fallbeispiel.

Es geschah am Ende einer Gruppensitzung, dass eine Patientin den Therapeuten plötzlich wüst beschimpfte. Sie warf ihm u.a. wütend vor, er sei doch überhaupt nicht an ihnen, den Patienten interessiert, sondern nur an der Sammlung von Daten für seine Karriere. (Die Gruppensitzungen wurden zu Forschungszwecken auf Tonband aufgezeichnet und die Patienten füllten u.a. Fragebögen nach den Gruppensitzungen aus). Sie, die Patienten, seien doch nur seine Datenlieferanten.

Erst in der Nachbesprechung der Sitzung mit der Gruppenschwester (die Behandlung fand in stationärem Rahmen statt) fiel dem Therapeuten ein, dass er während der Sitzung kurz, vermutlich nicht länger als ein oder zwei Minuten, aus dem Fenster geschaut und dabei an einen bevorstehenden Besprechungstermin mit einem Kollegen über ein Forschungsvorhaben gedacht hatte.

Die Reaktion der Patientin verdeutlicht: Sie hatte richtig wahrgenommen, dass der Therapeut mit seiner Aufmerksamkeit kurz nicht bei ihr bzw. der Gruppe war.

Aufgrund solcher klinischen Erfahrungen empfehlen wir TherapeutInnen, bei heftigen, scheinbar anlasslosen und die therapeutische Beziehung infrage stellenden Reaktionen von Borderline-PatientInnen nach einem realen Auslöser für diese Reaktionen zu suchen. Dieser hat - wie in obigem Beispiel - nach unserer Erfahrung häufig die Qualität und die Funktion eines Triggers und ist deshalb nicht einfach $\mathrm{zu}$ erkennen.

Werden aber Trigger als solche erkannt, ermöglicht das dem/r TherapeutIn in der Regel, sich wieder den Erfahrungen und dem Erleben des/r PatientIn positiv beachtend zuzuwenden. Er/sie kann dann verstehen, was die Reaktion des/r PatientIn ausgelöst hat.

\section{Der therapeutische Rahmen (Versorgungsaspekte)}

Behandlungsergebnisse und der erforderliche zeitliche Behandlungsaufwand

Ein/e PsychotherapeutIn sollte, bevor er/sie eine Behandlung mit einem/r PatientIn mit einer BorderlinePersönlichkeitsstörung aufnimmt, klären, ob er/sie in der Lage ist, dieses Behandlungsangebot mindesten drei bis fünf Jahre aufrecht zu erhalten. Das ist der für die meisten dieser PatientInnen erforderliche Zeitraum, um ihre Symptomatik soweit zu mildern, dass sie keine weitere kontinuierliche klinische Behandlung mehr brauchen.

Sechs Jahre nach der Eingangsdiagnostik werden rund $70 \%$ der PatientInnen, bei denen eine Borderline-Persönlichkeitsstörung diagnostiziert wurde, als remittiert eingestuft (Zanarini et al. 2005).

Die borderline-spezifische Symptomatik bildet sich auch in den dann folgenden Jahren weiter zurück, wenn auch nicht in allen Bereichen und auch nicht in gleichem Maße. In einer 10-Jahres-Katamnese stellten Zanarini et al. (2007) bei 290 PatientInnen fest, dass fast alle der 24 in der Eingangsdiagostik festgestellten borderline-spezifischen Symptome remittiert waren. Nur $40 \%$ der PatientInnen hatten auch 10 Jahre nach der Eingangsdiagnose weiterhin Probleme mit chronischen Ärger- und Wutgefühlen und ca. $20 \%$ Probleme mit dem Alleinsein sowie mit chronischen Abhängigkeitsgefühlen und masochistischen Tendenzen in Beziehungen.

Unsere eigenen Untersuchungen (s. Eckert und Biermann-Ratjen 2011) und unsere Erfahrungen mit 10-Jahres-Katmnesen stimmen mit diesen Ergebnissen gut überein. Wenn nach 10 Jahren noch Symptome vorlagen bzw. beklagt wurden, dann folgte daraus aber keine weitere Behandlungsnotwendigkeit.

Langzeitkatamnesen weisen darauf hin, dass die Lebensqualität der ehemaligen PatientInnen beeinträchtigt zu bleiben scheint (Zeitler et al. 2018). Bei unseren eigenen Nachuntersuchungen fiel uns auf, dass sehr viele der von uns nachuntersuchten Patient- 
Innen alleine lebten oder, wenn sie in einer Paarbeziehung lebten, in der Regel keine Kinder hatten. Unmittelbare Rückschlüsse auf die Lebensqualität lässt das u.E. aber nicht zu. Eine Patientin begründete ihre Kinderlosigkeit als bewussten Verzicht. Sie habe ja am eigenen Leibe erfahren, dass nicht jede Mutter eine gute Mutter für ihre Kinder sein könne.

Die Patientin, deren ausführliche Falldarstellung in Dulz und Eckert (2007, S. 589-593) nachzulesen ist, hatte, nachdem sie ihre Diagnose erfahren hatte, ängstlich gefragt: Einmal Borderlinerin immer Borderlinerin? Auf Grund unseres heutigen Wissens können wir ihr versichern, dass diese Diagnose keine lebenslange ist.

\section{Behandlungsfrequenz}

Die Behandlungsfrequenz hängt u. a. davon ab, ob die Behandlung stationär oder ambulant durchgeführt wird.

Im stationären Rahmen sind vier wöchentliche Therapiesitzungen (Gruppentherapie) die Regel. Hin$\mathrm{zu}$ kommen weitere therapeutische Angebote, wie Kunsttherapie oder gruppendynamische Übungen oder gemeinsame Unternehmungen aller PatientInnen einer Psychotherapiestation außerhalb der Klinik, wie Ausflüge oder Museumsbesuche.

Im ambulanten Setting haben sich sowohl für die Einzel- als auch für die Gruppentherapie zwei Sitzungen pro Woche bewährt.

\section{Behandlungsunterbrechungen}

Nimmt ein/e PatientIn mit einer Borderline-Persönlichkeitsstörung einen Behandlungstermin ohne Absage nicht wahr und meldet sich auch nicht vor dem nächsten Termin, empfehlen wir, ihn/sie zu kontaktieren, um den Grund für sein Nichterscheinen zu erfahren und zu vermitteln, dass wir uns wünschen, dass er/sie den nächsten möglichen Termin wieder wahrnimmt.

Hintergrund dieser Empfehlung ist die Schwierigkeit von Borderline-PatientInnen, Beziehungen realitätsgerecht „innerlich festzuhalten“. Versäumt ein/e PatientIn eine Therapiesitzung, kann bei ihm rasch die Befürchtung Oberhand gewinnen, dass der/die TherapeutIn sein Fernbleiben als Entwertung verbucht und ihn/sie deshalb nicht mehr sehen will bzw. die Behandlung für beendet erklärt.

\section{Behandlungsabschluss}

Der Behandlungsabschluss muss für PatientInnen mit einer Borderline-Persönlichkeitsstörung wegen der störungsbedingten Trennungsproblematik gut vorbereitet werden.

Im stationären Rahmen konnten wir die Probleme bei der Entlassung deutlich reduzieren, indem nicht mehr der/die einzelne PatientIn nach den vorgesehenen drei Monaten die Station verlassen musste, sondern alle PatientInnen einer Therapiegruppe zum selben Zeitpunkt entlassen wurden, weil wir das Grup- penformat auf geschlossene Gruppen umgestellt hatten.

Hilfreich im stationären Setting war auch das Angebot einer ambulanten Nachbehandlungsgruppe, die für jeden ein Jahr lang offen stand.

Im ambulanten Setting hilft das Angebot des/der TherapeutIn, dass sich der/die PatientIn bei Bedarf wieder an ihn wenden kann. Erfahrungsgemäß gehen PatientInnen mit diesem Angebot sehr „sparsam“ um. Nicht selten genügt es, dass der/die TherapeutIn telefonisch erreicht wird und versichert, dass dieses Angebot weiterhin besteht.

\section{Parallele Behandlungen}

Nach unserer klinischen Erfahrung ist es besser in den Fällen, in denen Borderline-PatientInnen neben der Gesprächspsychotherapie weitere Behandlungen haben, diese nicht generell - z. B. als Ausdruck von „Agieren“ - zu verbieten.

Viele PatientInnen haben die Erfahrung machen müssen, dass ihre Mütter auf den natürlichen frühkindlichen Entwicklungsschritt der Trennung bzw. in die Selbständigkeit inadäquat reagiert haben. TherapeutInnen sollten deshalb ihren PatientInnen ermöglichen, sich auch anderswo nach Hilfe und Unterstützung umzusehen. Wenn sie das Aufsuchen eines/r weiteren BehandlerIn als unausgesprochene Kritik an ihren therapeutischen Fähigkeiten erleben, ist das nach Möglichkeit zum Thema in der Therapiesitzung zu machen. Generell sollten TherapeutInnen PatientInnen mit weiteren Behandlungen ermuntern, von den Erfahrungen, die sie dabei machen, dann, wenn das für sie wichtig ist, zu berichten.

\section{Personale Behandlungskontinuität als Behandlungsprinzip}

Personale Behandlungskontinuität scheint besonders bei PatientInnen mit einer Borderline-Persönlichkeitsstörung für einen erfolgreichen Behandlungsverlauf bedeutsam zu sein.

Auch deshalb sollte der/die TherapeutIn einen Behandlungszeitraum von mindesten 3-5 Jahren für die Behandlung eines/r Borderline-PatientIn einplanen.

Wenn es in einer ambulanten Behandlung zu Krisen kommt, die eine stationäre Aufnahme zur Folge haben, sollte der/die ambulante BehandlerIn nach Möglichkeit während des stationären Aufenthaltes den Kontakt zu ihm/r aufrecht erhalten und die Wiederaufnahme der ambulanten Behandlung vorbereiten.

\section{Sollte jede/r Gesprächspsychotherapeutln auch PatientInnen mit Borderline-Persönlichkeitsstörungen behandeln?}

Jede/r PsychotherapeutIn macht im Verlauf der Berufstätigkeit die Erfahrung, dass er/sie bei manchen PatientInnen „erfolgreicher“ ist als bei anderen. Das hängt von verschieden Dingen ab. Manchmal sind es bestimmte Störungen, z. B. Abhängigkeitserkrankun- 
gen, mit denen er weniger gut zurechtkommt, manchmal Eigenschaften der Person, wie die ältere Person, die seiner Mutter/seinem Vater so ähnlich ist.

In solchen Fällen ist zu befürchten, dass der/die TherapeutIn das gesprächspsychotherapeutische Beziehungsangebot nicht aufrechterhalten kann. Er/sie kann den Patienten nicht bedingungsfrei positiv beachten und sich dessen auch nicht bewusst werden, d.h. er kann nicht kongruent sein.

Das heißt: Nicht jede/r GesprächspsychotherapeutIn sollte auch Borderline-PatientInnen behandeln. Er/sie sollte aber so gut mit KollegInnen und Einrichtungen „vernetzt“ sein, dass er/sie einem/r PatientIn mit einer Borderline-Persönlichkeitsstörung raten kann, wohin bzw. an wen er/sie sich mit seinem/ihrem Hilfeersuchen bzw. Behandlungswunsch wenden kann.

Interessenkonflikt J. Eckert und E.-M. Biermann-Ratjen geben an, dass kein Interessenkonflikt besteht.

Open Access Dieser Artikel wird unter der Creative Commons Namensnennung 4.0 International Lizenz veröffentlicht, welche die Nutzung, Vervielfältigung, Bearbeitung, Verbreitung und Wiedergabe in jeglichem Medium und Format erlaubt, sofern Sie den/die ursprünglichen Autor(en) und die Quelle ordnungsgemäß nennen, einen Link zur Creative Commons Lizenz beifügen und angeben, ob Änderungen vorgenommen wurden.

Die in diesem Artikel enthaltenen Bilder und sonstiges Drittmaterial unterliegen ebenfalls der genannten Creative Commons Lizenz, sofern sich aus der Abbildungslegende nichts anderes ergibt. Sofern das betreffende Material nicht unter der genannten Creative Commons Lizenz steht und die betreffende Handlung nicht nach gesetzlichen Vorschriften erlaubt ist, ist für die oben aufgeführten Weiterverwendungen des Materials die Einwilligung des jeweiligen Rechteinhabers einzuholen.

Weitere Details zur Lizenz entnehmen Sie bitte der Lizenzinformation auf http://creativecommons.org/licenses/by/4. 0/deed.de.

\section{Literatur}

Biermann-Ratjen, E.-M., Eckert, J., \& Gahleitner, S. B. (2019). Die gesprächspsychotherapeutische Behandlung von Traumafolgestörungen. In H. G. Seidler, H. J. Freyberger, H. Glaesmer \& S.B. Gahleitner (Hrsg.), Handbuch der Psychotraumatologie (Bd. 3, S. 859-876). Stuttgart: KlettCotta.

Biermann-Ratjen, E.-M., Eckert, J., \& Schwartz, H.-J. (2016). Gesprächspsychotherapie. Verändern durch Verstehen (10.Aufl.).Stuttgart:Kohlhammer.
Dulz, B., \&Eckert,J. (2007). Psychotherapiebei Borderline-Persönlichkeitsstörungen. In C. Reimer, J.Eckert, M. Hautzinger \& E. Wilke (Hrsg.), Psychotherapie. Ein Lehrbuch für Ärzte und Psychologen (Bd. 3, S. 565-594). Berlin, Heidelberg, New York: Springer.

Eckert, J. (2018). Borderline-Persönlichkeitsstörungen. In G. Stumm \& W.W. Keil (Hrsg.), Praxis der Personzentrierten Psychotherapie (2. Aufl. S. 237-246). Berlin, Heidelberg, NewYork: Springer.

Eckert, J., \& Biermann-Ratjen, E.-M. (2011). Gesprächspsychotherapie. In B. Dulz, S. Herpertz, O.F. Kernberg \& U. Sachsse (Hrsg.), Handbuch der Borderlinestörungen (2.Aufl.S.681-692). Stuttgart: Schattauer.

Eckert, J., Biermann-Ratjen, E.-M., \& Höger, D. (2012). Gesprächspsychotherapie. Lehrbuch (2. Aufl.). Berlin, Heidelberg, New York: Springer.

Eckert, J., Rohde-Dachser, C., \& Brodbeck, D. (2005). Diagnostisches Interview für Borderline-Patienten. Revidierte Fassung. In B. Strauß \& J. Schumacher (Hrsg.), Klinische Interviews und Ratingskalen (S.111-115). Göttingen: Hogrefe.

Falkai, P., \& Wittchen, H.-U. (Hrsg.). (2018). Diagnostisches und Statistisches Manual Psychischer Störungen DSM-5: Deutsche Ausgabe (2. Aufl.). Göttingen:Hogrefe.

Rogers, C. R. (1959). A theory of therapy, personality, and interpersonal relationships, as developed in the client-centered framework. In S. Koch (Hrsg.), Psychology: a study of a science (Bd. 3, S. 184-256). New York: McGraw-Hill. Deutsch: Rogers, C. R. (2009), Eine Theorie der Psychotherapie, der Persönlichkeit und der zwischenmenschlichen Beziehungen. Entwickelt im Rahmen des klientenzentrierten Ansatzes. München: Ernst Reinhardt.

Rohde-Dachser, C. (1995). Das Borderline-Syndrom (5. Aufl.). Bern:Huber.

Stumm, G., \& Keil, W.W. (Hrsg.). (2018). Praxis der Personzentrierten Psychotherapie (2. Aufl.). Berlin, Heidelberg, New York: Springer.

Zanarini, M.C., Frankenburg, F.R., Bradford Reich, D., Silk, K. R., Hudson, J. I., \& McSweeney, L. B. (2007). The Subsyndromal phenomenology of borderline personality disorder: a 10-year follow-up study. Am J Psychiatry, 164, 929-935.

Zanarini, M. C., Frankenburg, F. R., Hennen, J., Reich, D. B., \& Silk, K. R. (2005). The McLean Study of Adult Development (MSAD): overview and implications of the first 6 years of prospective follow-up. J Personal Disord, 19, 505-523.

Zeitler, M.-L., Bohus, M., Kleindienst, N., Knies, R., Ostermann, M., Schmahl, C., \& Lyssenko, L. (2018). How to assess recovery in borderline personality disorder: psychosocial functioning and satisfaction with life in a sample offormer DBT study patients. Journal of Personality Disorders, 34, 289-307.

Hinweis des Verlags Der Verlag bleibt in Hinblick auf geografische Zuordnungen und Gebietsbezeichnungen in veröffentlichten Karten und Institutsadressen neutral. 